\title{
A LOCAL-GLOBAL PRINCIPLE FOR REPRESENTATIONS OF BINARY FORMS BY CERTAIN QUINARY FORMS
}

\author{
Myung-Hwan Kim AND ByeOng-KweON OH
}

\begin{abstract}
In this article, we prove a certain local-global principle for representation of binary forms by an infinite family of quinary positive integral quadratic forms.
\end{abstract}

\section{Introduction}

One of the most fundamental questions in representation theory of integral quadratic forms is about the local-global principle, which is as follows:

Let $M$ and $N$ be positive integral quadratic forms of rank $m$ and $n$, respectively, such that $M$ represents $N$ locally at every prime spot. Under what condition does $M$ represent $N$ globally?

Concerning this question, we let $\mathfrak{R}_{m}(n)$ be the set of all positive integral quadratic forms $M$ of rank $m$ satisfying the following property:

$(\mathfrak{R}) M$ represents all positive integral quadratic forms $N$ of rank $n$ provided that $N \rightarrow M$ over $\mathbb{Z}_{p}$ at all $p$ and $\min (N)>C(M)$ for some positive constant $C(M)$ depending only on $M$ and $n$.

We define $R(n)$ to be the minimum rank $m$ for which $\Re_{m}(n)$ equals the set of all positive integral quadratic forms of rank $m$. In 1929 Tartakowsky [22] proved

$$
R(1)=5
$$

Received August 14, 2001

2000 Mathematics Subject Classification: 11E12, $11 \mathrm{H} 06$.

Key words and phrases: local-global principle, representation of quadratic forms.

This work was partially supported by KOSEF (99-0701-01-5-2). 
and in 1978, J. S. Hsia, Y. Kitaoka and M. Kneser [5] made a breakthrough by proving that

$$
R(n) \leq 2 n+3
$$

On the other hand, Y. Kitaoka [9] gave examples of positive integral quadratic forms of rank $n+3$ that are not contained in $\mathfrak{R}_{n+3}(n)$. Therefore, we have

$$
n+4 \leq R(n) \leq 2 n+3 .
$$

Recently, M. Jöchner [8] proved

$$
R(2)=6 .
$$

See [8] for other interesting results in this direction.

As appeared in Kitaoka's examples, the primitiveness condition on local representations seems to play an important role in studying the local-global principle for representations of integral quadratic forms. Regarding this, we define $\mathfrak{R}_{m}^{*}(n)$ to be the set of all positive integral quadratic forms $M$ of rank $m$ satisfying the following property:

$\left(\mathfrak{R}^{*}\right) M$ represents all positive integral quadratic forms $N$ of rank $n$ provided that $N \rightarrow M$ primitively over $\mathbb{Z}_{p}$ at all $p$ and $\min (N)>C^{*}(M)$ for some positive constant $C^{*}(M)$ depending only on $M$ and $n$.

We define $R^{*}(n)$ to be the minimum rank $m$ for which $\mathfrak{R}_{m}^{*}(n)$ equals the set of all positive integral quadratic forms of rank $m$. It is clear that

$$
\mathfrak{R}_{m}(n) \subseteq \mathfrak{R}_{m}^{*}(n) \text { and } R^{*}(n) \leq R(n) .
$$

In $[10,11]$, Kitaoka proved that

$$
\mathfrak{R}_{2 n+2}^{*}(n)=\mathfrak{R}_{2 n+2}(n) \text { for } n \geq 2
$$

and

$$
\mathfrak{R}_{2 n+1}^{*}(n)=\mathfrak{R}_{2 n+1}(n) \text { for } n \geq 3 .
$$

It is well known that

$$
R^{*}(1)=4
$$

(see [1] and [4]) and from the recent result of [8] follows that

$$
5 \leq R^{*}(2) \leq 6 .
$$


In this paper, we find an infinite family of quinary positive integral quadratic forms that is contained in $\mathfrak{R}_{5}^{*}(2)$. More precisely, we prove that every quinary positive even (or odd) integral quadratic form with even (or odd, respectively) squarefree discriminant that contains a quaternary sublattice of class number 1 as an orthogonal direct summand is contained in $\mathfrak{R}_{5}^{*}(2)$. Furthermore, an explicit estimation of the constant $C^{*}(M)$ is provided. See [3] and [23] for their estimation of $C(M)$ when $n=1$, and $[2,12-18]$ for recent results of authors on representations of integral quadratic forms related to the local-global principle.

We shall adopt lattice theoretic language. A $\mathbb{Z}$-lattice $L$ is a finitely generated free $\mathbb{Z}$-module in $\mathbb{R}^{n}$ equipped with a non-degenerate symmetric bilinear form $B$ such that $B(L, L) \subseteq \mathbb{Z}$. The corresponding quadratic map is denoted by $Q$.

For a $\mathbb{Z}$-lattice $L$ with basis vectors $\mathbf{e}_{1}, \mathbf{e}_{2}, \cdots, \mathbf{e}_{n}$, i.e., $L=\mathbb{Z} \mathbf{e}_{1}+$ $\mathbb{Z} \mathbf{e}_{2}+\cdots+\mathbb{Z} \mathbf{e}_{n}$, we often write

$$
L=\left(B\left(\mathbf{e}_{i}, \mathbf{e}_{j}\right)\right) .
$$

For sublattices $L_{1}, L_{2}$ of $L, L=L_{1} \perp L_{2}$ means $L=L_{1} \oplus L_{2}$ and $B\left(\mathbf{v}_{1}, \mathbf{v}_{2}\right)=0$ for all $\mathbf{v}_{1} \in L_{1}, \mathbf{v}_{2} \in L_{2}$. We call $L$ diagonal if it admits an orthogonal basis and in this case, we simply write

$$
L=\left\langle Q\left(\mathbf{e}_{1}\right), Q\left(\mathbf{e}_{2}\right), \cdots, Q\left(\mathbf{e}_{n}\right)\right\rangle,
$$

where $\left\{\mathbf{e}_{1}, \mathbf{e}_{2}, \cdots, \mathbf{e}_{n}\right\}$ is an orthogonal basis of $L$. We call $L$ nondiagonal otherwise. $L$ is called positive definite or simply positive if $Q(\mathbf{e})>0$ for any $\mathbf{e} \in L, \mathbf{e} \neq \mathbf{0}$. As usual,

$$
d L=\operatorname{det}\left(B\left(\mathbf{e}_{i}, \mathbf{e}_{j}\right)\right)
$$

is called the discriminant of $L$. A $\mathbb{Z}$-lattice (or $\mathbb{Z}_{2}$-lattice) $L$ is called even when $Q(L) \subseteq 2 \mathbb{Z}$ (or $\subseteq 2 \mathbb{Z}_{2}$, respectively) and odd otherwise. Note that every even lattice with odd rank has even discriminant.

We define $R L:=R \otimes_{\mathbb{Z}} L$ for any commutative ring $R$ containing $\mathbb{Z}$. For a $\mathbb{Z}$-lattice $L$ and a prime $p$, we define

$$
L_{p}:=\mathbb{Z}_{p} L
$$

and call it the localization of $L$ at $p$. If $\left\{\mathbf{e}_{1}, \mathbf{e}_{2}, \cdots, \mathbf{e}_{n}\right\}$ is an orthogonal basis of the quadratic space $V=\mathbb{Q} L$ or $\mathbb{Q}_{p} L$, we write in short

$$
V=\left(Q\left(\mathbf{e}_{1}\right), Q\left(\mathbf{e}_{2}\right), \cdots, Q\left(\mathbf{e}_{n}\right)\right) .
$$


Let $\ell$ and $L$ be $\mathbb{Z}$-lattices (or $\mathbb{Z}_{p}$-lattices). We say $L$ represents $\ell$ and write

$$
\ell \rightarrow L
$$

if there is an injective $\mathbb{Z}$-linear (or $\mathbb{Z}_{p}$-linear, respectively) map $\sigma$ from $\ell$ into $L$ that preserves the bilinear forms. Such a map is called a representation. A representation $\sigma: \ell \rightarrow L$ is called a primitive representation if $\sigma(\ell)$ is a direct summand of $L$. We say that $L$ primitively represents $\ell$ and write

$$
\ell \rightarrow^{*} L
$$

if there exists a primitive representation from $\ell$ to $L$.

We define

$$
[a, b, c]:=\left(\begin{array}{cc}
a & b \\
b & c
\end{array}\right)
$$

for convenience. For unexplained terminology, notation, and basic facts about global or local lattices, we refer the readers to O'Meara [19].

\section{Primitive representations over $\mathbb{Z}_{p}$}

Let $L$ be a $\mathbb{Z}$-lattice and $\mathfrak{a}_{(p)} \subseteq \mathbb{Z}_{p}$ be an ideal. By abuse of terminology, we say that $L$ is $\mathfrak{a}_{(p)}$-maximal if $L_{p}$ is an $\mathfrak{a}_{(p)}$-maximal $\mathbb{Z}_{p}$-lattice. Note that for an ideal $\mathfrak{a} \subseteq \mathbb{Z}, L$ is $\mathfrak{a}$-maximal if and only if $L$ is $\mathfrak{a}_{p^{-}}$ maximal for all prime $p$ (see [19]).

Let $\ell$ be a binary $\mathbb{Z}_{2}$-maximal $\mathbb{Z}$-lattice. Then $\ell_{2}$ is isometric to one of the following 16 binary $\mathbb{Z}_{2}$-lattices:

$$
\langle 1, \alpha\rangle,\langle 3, \beta\rangle, \quad\langle\alpha, 2\rangle, \quad\langle\beta, 2 \gamma\rangle, \quad[2,1,2], \quad[0,1,0]
$$

where $\alpha=1,3,5,7, \beta=3,7$, and $\gamma=5,7$. The next two lemmas are useful in the proof of our main theorem in Section 3.

LEMMA 2.1. For an odd prime $p$, let $L_{(p)}=L_{(p)}^{\prime} \perp\langle p \eta\rangle$ be a quinary $\mathbb{Z}_{p}$-lattice with $d L_{(p)} \in p \mathbb{Z}_{p}^{*}$ and let $\ell_{(p)}=\left\langle\delta_{1} p^{u_{1}}, \delta_{2} p^{u_{2}}\right\rangle$ be a (binary) $\mathbb{Z}_{p}$-lattice with $\delta_{i} \in \mathbb{Z}_{p}^{*}, u_{1} \leq u_{2}$. Then $\ell_{(p)}$ cannot be primitively represented by $L_{(p)}$ if and only if $L_{(p)}^{\prime}$ has a nonsquare discriminant, $u_{1}$ is an odd integer less than $u_{2}$, and $\delta_{1} \eta \notin \mathbb{Z}_{p}^{* 2}$. 
Proof. The necessity is straightforward. For the sufficiency, suppose that $\ell_{(p)}$ is primitively represented by $L_{(p)}$. Since $\left\langle\delta_{2} p^{u_{2}}\right\rangle$ is primitively represented by $L_{(p)}$, it is also primitively represented by the orthogonal complement $K_{(p)}$ of $\left\langle\delta_{1} p^{u_{1}}\right\rangle$ in $L_{(p)}$. From Hasse symbol computation it follows that $\mathbb{Q}_{p} K_{(p)}$ is anisotropic and that the $p$-order of the scale of each modular component of the Jordan decomposition of $K_{(p)}$ is less than $u_{2}$, which is impossible.

Let $K_{(2)}$ be a quinary (odd) unimodular $\mathbb{Z}_{2}$-lattice or even $\mathbb{Z}_{2}$-lattice whose Jordan decomposition has even unimodular component of rank 4 and $2 \mathbb{Z}_{2}$-modular component of rank 1 . When $K_{(2)}$ is even, one can easily check that $K_{(2)}$ primitively represents all binary $\mathbb{Z}_{2}$-lattices. For the odd case, we have the following:

Lemma 2.2. Let $K_{(2)}$ be a quinary unimodular $\mathbb{Z}_{2}$-lattice. Then $K_{(2)}$ primitively represents all binary $\mathbb{Z}_{2}$-lattices unless

$$
K_{(2)} \simeq\left\langle 1,1,1,1, d K_{(2)}\right\rangle
$$

In the exceptional case, $K_{(2)}$ primitively represents all binary $\mathbb{Z}_{2}$-lattices but

$$
[4 \alpha, 2 \beta, 4 \gamma] \text { and }\left\langle d K_{(2)}\right\rangle \perp\langle 8 \delta\rangle,
$$

where $\alpha, \beta, \gamma, \delta \in \mathbb{Z}_{2}$. 7].

Proof. This is a direct consequence of Hensel's Lemma. See also [6,

\section{Quaternary sublattices of class number 1}

In this section, we assume that every $\mathbb{Z}$-lattice is positive unless stated otherwise.

For a binary $\mathbb{Z}$-lattice $\ell$, if $\ell=[a, b, c], 0 \leq 2 b \leq a \leq c$, for some basis $\left\{\mathbf{e}_{1}, \mathbf{e}_{2}\right\}$, which always exists, we say that $[a, b, c]$ is Minkowski reduced and that $\left\{\mathbf{e}_{1}, \mathbf{e}_{2}\right\}$ is a Minkowski reduced basis.

For an odd prime $p$, we denote a nonsquare unit in $\mathbb{Z}_{p}$ by $\Delta_{p}$. For two elements $\alpha, \beta \in \mathbb{Z}_{p}$, we write $\alpha \sim \beta$ if $\alpha \beta^{-1} \in \mathbb{Z}_{p}^{* 2}$. 
Let $L$ be a quaternary $\mathbb{Z}$-lattice with class number 1 . We partition all odd primes into the following three sets:

$$
\begin{aligned}
& W=\left\{p \mid L_{p} \text { is not unimodular }\right\} \\
& U=\left\{p \mid L_{p} \text { is unimodular with } d\left(L_{p}\right)=1\right\} \\
& V=\left\{p \mid L_{p} \text { is unimodular with } d\left(L_{p}\right)=\Delta_{p}\right\}
\end{aligned}
$$

For a positive integer $k$, we define

$$
L(\delta k):=L \perp\langle\delta k\rangle,
$$

where $\delta=1$ if $L$ is odd, and $\delta=2$ if $L$ is even. Let $V_{0}$ be the set of all odd prime divisors of $k$. We define $V_{1}:=V \cap V_{0}$ and $V_{2}:=V \backslash V_{1}$.

Lemma 3.1. Let $p>3$ be a prime and $a, b, c \in \mathbb{F}_{p}$. If $b^{2}-a c \not \equiv 0$ $(\bmod p)$, then the set $\left\{a x^{2}+2 b x+c \mid x \in \mathbb{F}_{p}\right\}$ contains both a nonzero square and a nonsquare.

Proof. See [21].

TheOREM 3.2. Assume that $d:=d L(2 k)$ is a squarefree integer. Let $\ell$ be a binary $\mathbb{Z}$-lattice such that

$$
\ell_{p} \rightarrow^{*} L(2 k)_{p}
$$

at all $p$. Then for any $\epsilon>0$, there exists a constant $C>0$ depending only on $\epsilon$ such that

$$
\text { if } \min (\ell)>C \cdot d^{5+\epsilon} \text {, then } \ell \rightarrow L(2 k) \text {. }
$$

Proof. Let $\ell=[a, b, c]$ be Minkowski reduced. We define

$$
\ell_{s}(t):=\left[a-2 k t^{2}, s a+b, s^{2} a+2 s b+c\right]=\left(\begin{array}{cc}
a-2 k t^{2} & s a+b \\
s a+b & s^{2} a+2 s b+c
\end{array}\right)
$$

where $s, t$ are integers. We assume for the time being that $a$ is large enough so that $\ell_{s}(t)$ is positive, and determine how large $a$ should be at the end of the proof. Since the class number of $L$ is 1 , if $\ell_{s}(t)_{p} \rightarrow L_{p}$ for all $p$ (including $\infty$ ), then $\ell_{s}(t) \rightarrow L$ and hence $\ell \cong \ell_{s}(0) \rightarrow L(2 k)$. We will find such $s$ and $t$ in the following. 
Note that for an odd prime $p$,

$$
\mathbb{Q}_{p}\left(\ell_{s}(t)\right) \rightarrow \mathbb{Q}_{p} L \text { if and only if } \ell_{s}(t)_{p} \rightarrow L_{p}
$$

(see [Theorem 2, 20]).

(a) $p \in U$ : In this case, $\ell_{s}(t)_{p} \rightarrow L_{p}$ for any integers $s$ and $t$.

(b) $p \in W \cup V_{1} \cup\{2\}$ : By the Chinese Remainder Theorem, it suffices to find integers $s$ and $t$ at each $p \in W \cup V_{1} \cup\{2\}$ such that $\ell_{s}(t)_{p} \rightarrow L_{p}$.

b-1) $p \in W$ : Let

$$
L_{p}=\langle 1,1, \delta, p \eta\rangle \text { and } \ell_{p}=\left[\alpha p^{u}, \beta p^{v}, \gamma p^{w}\right]
$$

where $\alpha, \beta, \gamma, \delta, \eta \in \mathbb{Z}_{p}^{*}$ and $u, v, w$ are nonnegative integers. Note that if $\operatorname{ord}_{p}\left(d \ell_{p}\right)$ is even or $d\left(\mathbb{Q}_{p} \ell\right)=-\delta \eta p \Delta_{p}$, then $\ell_{p} \rightarrow L_{p}$.

If $d \ell \neq \equiv 0(\bmod p)$, then any $s, t$ satisfying $t \equiv 0(\bmod p)$ can be taken so that $\ell_{s}(t)_{p}$ is unimodular, which implies $\ell_{s}(t)_{p} \rightarrow L_{p}$. Let $d \ell \equiv 0(\bmod p)$. Then take $s, t$ satisfying $s \equiv 0(\bmod p), t \not \equiv 0(\bmod p)$ if $u=v=w=0, d \ell \equiv 0(\bmod p)$; take $s, t$ satisfying $s t \not \equiv 0(\bmod p)$ if $u=0, v, w \neq 0$; and take $s, t$ satisfying $s t \not \equiv 0(\bmod p)$ if $u, v \neq 0, w=0$, so that $\ell_{s}(t)_{p}$ is unimodular, which again implies $\ell_{s}(t)_{p} \rightarrow L_{p}$.

It only remains to treat the case when $u, v, w \neq 0$. If $2 k \sim \delta$, then $\ell_{s}(t)_{p} \rightarrow L_{p}$ by taking any $s$ and $t$ satisfying $t \neq 0(\bmod p)$. So, we may assume that $2 k \sim \delta \Delta_{p}$.

Let $u<v, w$. In this case, we take $s, t$ satisfying $s, t \not \equiv 0(\bmod p)$. If $u$ is even, then $\ell_{s}(t)_{p} \rightarrow L_{p}$ follows immediately. Let $u$ be odd. Since

$$
\ell_{p} \simeq\left\langle\alpha p^{u}, \alpha\left(p^{w} \alpha \gamma-p^{2 v-u} \beta^{2}\right)\right\rangle
$$

we have $\alpha \sim \eta$ by Lemma 2.1, and hence

$$
d\left(\ell_{s}(t)_{p}\right) \sim-2 k p^{u} \alpha \sim-p^{u} \eta \delta \Delta_{p} .
$$

This implies $\ell_{s}(t)_{p} \rightarrow L_{p}$.

Let $u=v<w$. Then

$$
d\left(\ell_{s}(t)_{p}\right)=p^{u}\left(p^{w} \alpha \gamma-p^{u} \beta^{2}-2 k t^{2}\left(s^{2} \alpha+2 s \beta+p^{w-u} \gamma\right)\right) .
$$

If $p \neq 3$, then take $s, t$ satisfying

$$
-2 k t^{2}\left(s^{2} \alpha+2 s \beta+p^{w-u} \gamma\right) \sim-\eta \delta \Delta_{p},
$$


which is possible by Lemma 3.1. Then $\ell_{s}(t)_{p} \rightarrow L_{p}$ follows immediately. Let $p=3$. If $u$ is even, then take $s, t$ satisfying $t \not \equiv 0(\bmod 3)$ and $s^{2} \alpha+$ $2 s \beta \not \equiv 0(\bmod 3)$ so that $\ell_{s}(t)_{3} \rightarrow L_{3}$. Let $u$ be odd. If $w, u, w-u$ are all bigger than 1 , take $s, t$ satisfying $t \neq \equiv 0(\bmod 3), \operatorname{ord}_{3}(s)=1$. Then $\operatorname{ord}_{3}\left(d\left(\ell_{s}(t)_{3}\right)\right)$ is even, from which follows $\ell_{s}(t)_{3} \rightarrow L_{3}$. The remaining possibilities can be handled in a similar manner and are omitted.

Let $u=w<v$. The proof of this case is almost identical to the above except when $p=3$ and $u$ is odd. We may assume that $\alpha \gamma \equiv 2(\bmod 3)$ because the set $\left\{s^{2}+1 \mid s \in \mathbb{F}_{3}\right\}$ contains both a nonzero square and a nonsquare in $\mathbb{F}_{3}$. Let $t$ be any integer which is not divisible by 3 . It is tedious but not difficult to find an integer $s(\bmod 9)$ for which $\operatorname{ord}_{3}\left(d\left(\ell_{s}(t)_{3}\right)\right)$ is even. This implies $\ell_{s}(t)_{3} \rightarrow L_{3}$.

Let $u=v=w$. Then

$$
d\left(\ell_{s}(t)_{p}\right)=p^{u}\left(p^{u}\left(\alpha \gamma-\beta^{2}\right)-2 k t^{2}\left(s^{2} \alpha+2 s \beta+\gamma\right)\right)
$$

and the proof of this case is also very similar to the above except when $p=3, u$ is odd and $\alpha \gamma-\beta^{2} \equiv 0(\bmod 3)$. Note that $\alpha \sim \eta$. If we take $s, t$ satisfying $t \not \equiv 0(\bmod 3), 2 s \beta+\gamma \equiv 0(\bmod 3)$, then $\ell_{s}(t)_{3} \cong$ $\left\langle-2 k, 3^{u} \alpha\right\rangle \rightarrow L_{3}$.

Let $v<u, w$. We take $s, t$ satisfying $s \sim 2 \beta \eta, t \not \equiv 0(\bmod p)$. Then

$$
\begin{aligned}
d\left(\ell_{s}(t)_{p}\right) & =p^{v}\left(p^{u+w-v}-p^{v} \beta^{2}-2 k t^{2}\left(s^{2} p^{u-v}+2 s \beta+p^{w-v} \gamma\right)\right) \\
& \sim-k \beta s p^{v} \sim-\delta \Delta_{p} \eta p^{v}
\end{aligned}
$$

The rest is trivial.

Let $v=w<u$. We take $s, t$ satisfying $-2 k t^{2}(2 s \beta+\gamma) \in \mathbb{Z}_{p}^{*}$. Then

$$
d\left(\ell_{s}(t)_{p}\right) \sim-2 k t^{2}(2 s \beta+\gamma) p^{v}
$$

and the rest is trivial.

Finally, let $w<u, v$. The proof of this case is exactly same as that of the case when $u<v, w$ and is omitted.

b-2) $p \in V_{1}$ : Let

$$
L_{p}=\left\langle 1,1,1, \Delta_{p}\right\rangle \text { and } \ell_{p}=\left[\alpha p^{u}, \beta p^{v}, \gamma p^{w}\right]
$$

where $\alpha, \beta, \gamma \in \mathbb{Z}_{p}^{*}$ and $u, v, w$ are nonnegative integers. Let $\tau=2 k / p$. Note that if $\operatorname{ord}_{p}\left(d \ell_{p}\right)$ is odd or $d\left(\mathbb{Q}_{p} \ell\right)=-1$, then $\ell_{p} \rightarrow L_{p}$. We only provide proofs for the cases when $1 \leq u=w<v$ and $1 \leq u=w=v$ 
because all other cases can be proved in a very similar manner as in b-1).

Let $1 \leq u=w<v$. We may assume that $u$ is odd because

$$
d\left(\ell_{s}(t)_{p}\right)=p^{u+1}\left(p^{u-1} \alpha \gamma-p^{2 v-u-1} \beta^{2}-\tau t^{2}\left(s^{2} \alpha+2 s p^{v-u} \beta+\gamma\right)\right) .
$$

If $u \neq 1$, then we may further assume that $p=3$ by Lemma 3.1. If $\alpha \gamma \equiv$ $1(\bmod 3)$, then one can easily find $s, t$ for which $d\left(\ell_{s}(t)_{3}\right)=-3^{u+1}$ and hence $\ell_{s}(t)_{3} \rightarrow L_{3}$. Let $\alpha \gamma \equiv-1(\bmod 3)$. Then we can take $s, t$ satisfying

$$
\operatorname{ord}_{3}\left(s^{2} \alpha+2 s 3^{v-u} \beta+\gamma\right)=1 \text { and } t \not \equiv 0 \quad(\bmod 3),
$$

from which $\ell_{s}(t)_{p} \rightarrow L_{p}$ follows immediately. Let $u=1$. If $\alpha \gamma \equiv-1$ $(\bmod p)$, take any $s$ and $t$ satisfying $t \equiv 0(\bmod p)$. Then $d\left(\ell_{s}(t)_{p}\right)=$ $-p^{2}$ and hence $\ell_{s}(t)_{p} \rightarrow L_{p}$. Assume that $t \not \equiv 0(\bmod p)$. If $\alpha \gamma-\tau \gamma t^{2} \equiv$ $-1(\bmod p)$, then $s$ satisfying $s \equiv 0(\bmod p)$ will do. So assume that

$$
\alpha \gamma \not \equiv-1 \quad \text { and } \quad \alpha \gamma-\tau \gamma t^{2} \not \equiv-1 \quad(\bmod p) .
$$

If $\alpha-\tau t^{2} \equiv 0(\bmod p)$, then $d\left(\ell_{s}(t)_{p}\right)=-p^{u+1}$ for all $s$ satisfying $s \not \equiv 0$ $(\bmod p)$ and hence $\ell_{s}(t)_{p} \rightarrow L_{p}$. Let $\alpha-\tau t^{2} \not \equiv 0(\bmod p)$. Then $p \neq 3$ and hence Lemma 3.1 can be applied to find $s$ such that $d\left(\ell_{s}(t)_{p}\right)=-p^{2}$. From this follows $\ell_{s}(t)_{p} \rightarrow L_{p}$.

Let $1 \leq u=v=w$. The proof is almost identical to the above except when $u=1$ and $\alpha \gamma-\beta^{2} \neq \equiv(\bmod p)$. In the exceptional case, we have

$$
\begin{aligned}
d\left(\ell_{s}(t)_{p}\right) & =p^{2}\left(\alpha \gamma-\beta^{2}-\tau t^{2}\left(\alpha s^{2}+2 s \beta+\gamma\right)\right) \\
& =p^{2}\left(\left(\alpha \gamma-\beta^{2}\right)\left(1-\alpha^{-1} \tau t^{2}\right)-\tau t^{2}(\alpha s+\beta)^{2} \alpha^{-1}\right) \\
& =p^{2}\left(-\alpha \tau t^{2} s^{2}-2 \tau \beta t^{2} s+\left(\alpha-\tau t^{2}\right) \gamma-\beta^{2}\right) .
\end{aligned}
$$

We take $t$ satisfying $t \neq \equiv 0(\bmod p)$. If $\alpha-\tau t^{2} \equiv 0(\bmod p)$, then take $s$ satisfying $\alpha s+\beta \not \equiv 0(\bmod p)$ so that $d\left(\ell_{s}(t)_{p}\right) \sim-p^{2} \alpha^{-1} \tau \sim-p^{2}$. Let $\alpha-\tau t^{2} \not \equiv 0(\bmod p)$. If $p \neq 3$, then because

$$
\left(\tau \beta t^{2}\right)^{2}-\left(-\alpha \tau t^{2}\right)\left(\left(\alpha-\tau t^{2}\right) \gamma-\beta^{2}\right)=\tau t^{2}\left(\alpha \gamma-\beta^{2}\right)\left(\alpha-\tau t^{2}\right) \not \equiv 0 \quad(\bmod p)
$$

we can apply Lemma 3.1 to conclude that $d\left(\ell_{s}(t)_{p}\right) \sim-p^{2}$. We may conclude the same even if $p=3$ from case by case consideration. In either case it follows that $\ell_{s}(t)_{p} \rightarrow L_{p}$. 
b-3) $p=2$ : Since $L_{2}$ is even unimodular $\mathbb{Z}_{2}$-lattice, $L_{2}$ is isometric to either

$$
[0,1,0] \perp[0,1,0] \quad \text { or } \quad[0,1,0] \perp[2,1,2] .
$$

Since the former represents all binary even $\mathbb{Z}_{2}$-lattices, assume that $L_{2}$ is isometric to the latter. Then $L_{2}$ represents all binary primitive even $\mathbb{Z}_{2}$-lattices except $[4,2,4]$. So any binary even $\mathbb{Z}_{2}$-lattice that represents a 2-adic integer $\theta$ with or $_{2}(\theta)=1$ is represented by $L_{2}$. Thus by taking any $s$ and $t$ satisfying $t \not \equiv a / 2(\bmod 2)$, we obtain $\ell_{s}(t)_{2} \rightarrow L_{2}$.

(c) $p \in V_{2}$ : Let $T$ be the product of those primes $p \in W \cup V_{1} \cup\{2\}$ for which we took $t$ satisfying $t \equiv 0(\bmod p)$ in order for $L_{p}$ to represent $\ell_{s}(t)_{p}$ in (b).

Note that every binary $\mathbb{Z}_{p}$-lattice whose scale is $\mathbb{Z}_{p}$ can be represented by $L_{p}$. It suffices to find a suitable integer $S$ such that $\operatorname{gcd}\left(a-2 k T^{2}, S a+\right.$ $b, p)=1$ so that $\ell_{S}(T)_{p} \rightarrow L_{p}$ for all $p \in V_{2}$. Let

$$
B:=3^{\nu} \times \prod_{p \in W \cup V_{1}} p
$$

where $\nu=1$ if $3 \in W \cup V_{1}$ and $\nu=0$ otherwise, and take $s^{\prime} \leq B$ such that $\ell_{s^{\prime}}(T)_{p} \rightarrow L_{p}$ for all $p \in W \cup V_{1} \cup\{2\}$. Then clearly, $\ell_{B h+s^{\prime}}(T)_{p} \rightarrow L_{p}$ for all integers $h$ and $p \in W \cup V_{1} \cup\{2\}$.

Let $A$ be the set of all primes dividing $a-2 k T^{2}$ and let

$$
A \cap V_{2}=\left\{q_{1}, q_{2}, \ldots, q_{e}\right\} .
$$

We may assume that $e \geq 1$. Note that $\operatorname{gcd}\left(B a, q_{1} q_{2} \cdots q_{e}\right)=1$. By Lemma 3 in [12], if we let $g$ be the smallest integer satisfying

$$
2 g+1 \geq\left(q_{1}+e-1\right) 2^{e} /\left(q_{1}-1\right)
$$

then there exists an integer $S$ in the set

$$
\left\{B(-g)+s^{\prime}, B(-g+1)+s^{\prime}, \ldots, s^{\prime}, \ldots, B(g-1)+s^{\prime}, B g+s^{\prime}\right\}
$$

such that $S a+b$ is not divisible by $q_{i}$ for all $i, 1 \leq i \leq e$. If we take this $S$, then $\ell_{S}(T)_{p} \rightarrow L_{p}$ for all $p \in V_{2}$.

Note that the with $S$ and $T$ chosen above, $\ell_{S}(T)_{p} \rightarrow L_{p}$ holds not only at all $p \in V_{2}$ but also at all $p \in W \cup V_{1} \cup\{2\} \cup U$. Furthermore, if

$$
\min (\ell)=a>3 d^{5}(g+2)^{2},
$$


then

$$
\begin{aligned}
d\left(\ell_{S}(T)\right) & =a c-b^{2}-2 k T^{2}\left(S^{2} a+2 S b+c\right) \\
& \geq \frac{a c}{4}-b^{2}+\frac{3 a c}{4}-2 k T^{2}\left(S^{2} c+|S| c+c\right) \\
& \geq \frac{3 a c}{4}-2 k T^{2}(|S|+1)^{2} c \\
& \geq \frac{3 c}{4}\left(a-\frac{8}{3} k T^{2} B^{2}(g+2)^{2}\right) \\
& \geq \frac{3 c}{4}\left(a-3 d^{5}(g+2)^{2}\right)>0,
\end{aligned}
$$

where the last line follows from $2 k \leq d, T \leq d$, and $2 B \leq 3 d$.

Note that $a>a-2 k T^{2} \geq q_{1} q_{2} \cdots q_{e}$ and that $3(g+2)^{2} \leq 12 e^{2} 4^{e}$. Let $e_{0}$ be the largest positive integer $e$ for which $12 e^{2} 4^{2} \geq 5^{e}$ and let $C_{0}:=12 e_{0}^{2} 4^{e_{0}}$. We assume that $3 \leq q_{1}<q_{2}<\cdots<q_{e}$.

Let any small enough $\epsilon>0$ be given. Choose a smallest prime $q$ such that $\epsilon \log _{5}(\sqrt{q} / 5)>5$. Let $q$ be the $j$-th smallest odd prime. Then define

$$
C=C(\epsilon):=\max \left\{C_{0}, 5^{2 j}\right\} .
$$

It suffices to show that $a>C(\epsilon) d^{5+\epsilon}$ implies $a>3 d^{5}(g+2)^{2}$.

If $e \leq e_{0}$, then

$$
a>C(\epsilon) d^{5+\epsilon}>C_{0} d^{5} \geq 12 e^{2} 4^{e} d^{5} \geq 3(g+2)^{2} d^{5} .
$$

So, we may assume $e>e_{0}$. If $e \leq 2 j$, then

$$
a>C(\epsilon) d^{5+\epsilon} \geq 5^{2 j} d^{5+\epsilon}>5^{\mathrm{e}} d^{5}>3(g+2)^{2} d^{5} .
$$

So, we may further assume $e>2 j$. Let $e \leq \epsilon \log _{5} d$. Then

$$
a>C(\epsilon) d^{5+\epsilon} \geq C(\epsilon) d^{5} 5^{e}>d^{5} 5^{e}>3 d^{5}(g+2)^{2} .
$$

Let $e>\epsilon \log _{5} d$. Note that $a>q^{e / 2}$ by the choice of $q$ and the assumption $e>2 j$. Then

$$
\frac{q^{e / 2}}{5^{e} d^{5}}=\left(\frac{\sqrt{q}}{5}\right)^{e} \cdot \frac{1}{d^{5}}>\left(\frac{\sqrt{q}}{5}\right)^{\epsilon \log _{5} d} \cdot \frac{1}{d^{5}}=d^{\epsilon \log _{5}(\sqrt{q} / 5)} \cdot \frac{1}{d^{5}}>1,
$$

which implies. $a>q^{e / 2}>5^{e} d^{5}>3 d^{5}(g+2)^{2}$ as desired. 
Therefore we can always choose a suitable positive constant $C=C(\epsilon)$ depending only on $\epsilon$ such that $\ell_{S}(T)$ is positive. This completes the proof.

Observe that the constant $C^{*}(M)$ introduced in $\left(\mathfrak{R}^{*}\right)$ is $C(\epsilon)(d M)^{5+\epsilon}$, which depends only on $M=L(2 k)$ and the $\operatorname{rank}(\ell)=2$.

REMARK 3.3. The primitiveness condition on local representations cannot be omitted in Theorem 3.2 (see [9]). For example, let

$$
\ell=\left\langle 140 \cdot 3^{2 m}, 30 \cdot 7^{2 m}\right\rangle, \quad L=[2,1,2] \perp[2,1,4] .
$$

For any integer $k>70$ satisfying $k \equiv 2(\bmod 21)$, suppose that $\ell \rightarrow$ $L(2 k)$. Then there exist integers $t$ and $r$ such that $\ell(t, r) \rightarrow L$, where

$$
\begin{aligned}
\ell(t, r): & =\left[140 \cdot 3^{2 m}-2 k t^{2},-2 k t r, 30 \cdot 7^{2 m}-2 k r^{2}\right] \\
& =\left(\begin{array}{cc}
140 \cdot 3^{2 m}-2 k t^{2} & -2 k t r \\
-2 k t r & 30 \cdot 7^{2 m}-2 k r^{2}
\end{array}\right) .
\end{aligned}
$$

But one can easily check that $\ell(t, r)_{3} \nrightarrow L_{3}$ if $t \neq 0$ or $t=r=0$ and that $(\ell(t, r))_{7} \nrightarrow L_{7}$ if $r \neq 0$ for all $m$. Note that $\ell_{p} \nrightarrow^{*} L(2 k)_{p}$ at $p=3,7$ although $\ell_{p} \rightarrow L(2 k)_{p}$ at all $p$.

We now turn to the odd version of Theorem 3.2.

THEOREM 3.4. Assume that $d:=d L(k)$ is an odd squarefree integer. Let $\ell$ be a binary $\mathbb{Z}$-lattice such that

$$
\ell_{p} \rightarrow^{*}(L(k))_{p}
$$

at all $p$. Then for any $\epsilon>0$, there exists a constant $C>0$ depending only on $\epsilon$ such that

$$
\text { if } \min (\ell)>C \cdot d^{5+\epsilon} \text {, then } \ell \rightarrow L(k) \text {. }
$$


Proof. Let $\ell=[a, b, c]$ be Minkowski reduced. We define

$$
\ell_{s}(t):=\left[a-k t^{2}, s a+b, s^{2} a+2 s b+c\right] .
$$

Since the proof is completely identical to that of Theorem 3.2 except at $p=2$, we only consider $p=2$. We will find integers $s$ and $t$ for which $\ell_{s}(t)_{2} \rightarrow L_{2}$. Then the theorem follows from replacing $T$ and $B$ in the previous theorem by

$$
T^{\prime}=2^{o r d_{2}(t)-o r d_{2}(T)} T \text { and } B^{\prime}=4 B .
$$

Since $L_{2}$ is quaternary odd unimodular $\mathbb{Z}_{2}$-lattice, $L_{2}$ represents all $\mathbb{Z}_{2}$-maximal $\mathbb{Z}_{2}$-lattices except the following cases:

\begin{tabular}{|c|c|c|c|c|}
\hline$L_{2}$ & exceptions & 1 & $L_{2}$ & exceptions \\
\hline$\langle 1,1,1,1\rangle$ & $\langle 1,7\rangle,[0,1,0]$ & 1 & $\langle 1,1,1,5\rangle$ & $\langle 1,3\rangle, \quad[0,1,0]$ \\
\hline$\langle 1,1,3,3\rangle$ & {$[2,1,2]$} & 1 & $\langle 1,1,3,7\rangle$ & {$[2,1,2]$} \\
\hline$\langle 1,1,1,3\rangle$ & $\langle 3,7\rangle$ & 1 & $\langle 1,1,1,7\rangle$ & $\langle 3,3\rangle$ \\
\hline$\langle 1,3,3,3\rangle$ & $\langle 1,5\rangle$ & 1 & $\langle 1,7,7,7\rangle$ & $\langle 1,1\rangle$. \\
\hline
\end{tabular}

Table 3.1

Note also that if $\operatorname{ord}_{2}\left(d\left(\mathbb{Q}_{2} \ell\right)\right)$ is odd, then $\ell_{2} \rightarrow L_{2}$.

(a) Firstly, we consider the case when $L(k)_{2}$ primitively represents all binary $\mathbb{Z}_{2}$-lattices. Since $S_{2}(L)=-(d L, d L \cdot k)$, we know that $L \not$ $\langle 1,1,1, \epsilon\rangle$, where $S_{2}(L)$ is the 2-adic Hasse symbol of $L$ and $\epsilon \equiv 1$ $(\bmod 4)$. If $\ell_{s}(t)_{2}$ is an odd lattice, then

$$
\ell_{s}(t)_{2} \rightarrow L_{2} \text { if and only if } \mathbb{Q}_{2}\left(\ell_{s}(t)\right) \rightarrow \mathbb{Q}_{2} L
$$

by Table 3.1. Furthermore, if $\ell_{s}(t)_{2}$ represents a unit $\eta \equiv-k(\bmod 4)$, then one can easily check that $\ell_{s}(t)_{2} \rightarrow L_{2}$. Let $\ell_{2}=\left[\alpha 2^{u}, \beta 2^{v}, \gamma 2^{w}\right]$, where $\alpha, \beta, \gamma \in \mathbb{Z}_{2}^{*}$. Note that

$$
d\left(\ell_{s}(t)_{2}\right)=2^{u+w} \alpha \gamma-2^{2 v}-k t^{2}\left(s^{2} 2^{u} \alpha+s 2^{v+1} \beta+2^{w} \gamma\right) .
$$


If $u \geq 2$, we take $t$ satisfying $\operatorname{ord}_{2}(t)=0$. Then $\left\langle 2^{u} \alpha-k t^{2}\right\rangle \rightarrow \ell_{s}(t)_{2}$, which implies $\ell_{s}(t)_{2} \rightarrow L_{2}$. If $u=1, v \geq 1, w \geq 2$, we take $s, t$ satisfying $s \equiv 1(\bmod 2), \operatorname{ord}_{2}(t)=0$ so that $\operatorname{ord}_{2}\left(d\left(\ell_{s}(t)_{2}\right)\right)=1$ and hence $\ell_{s}(t)_{2} \rightarrow L_{2}$. Since the remaining subcases can be treated in a very similar manner, we only list the choices of $s$ and $t$ for all possible subcases in Table 3.2 after the proof.

(b) We now treat the other case. If $u \geq 2, w \geq 2$, then $v=0$ by the primitiveness of representation. Consider the two choices of $s, t: s \equiv 0$ $(\bmod 4), \operatorname{ord}_{2}(t)=0$ and $s \equiv 2(\bmod 4), \operatorname{ord}_{2}(t)=0$. Both make $\ell_{s}(t)_{2}$ odd unimodular but yield different discriminants. Therefore by Table 3.1 , one of the two should be represented by $L_{2}$. Let $u \geq 3, v \geq 2, w=0$. Since $\gamma \not \equiv d L \cdot k(\bmod 8)$ by the primitiveness condition, if we take $\operatorname{ord}_{2}(t)=0$, then $\ell_{s}(t)_{2}$ is odd unimodular and $d\left(\ell_{s}(t)_{2}\right) \not \equiv-d\left(L_{2}\right)$. This implies that $\ell_{s}(t)_{2} \rightarrow L_{2}$. Now let $u=0, v \geq 2, w=2$. If we take $s \equiv 0(\bmod 4)$, ord $_{2}(t)=1$, then $d\left(\ell_{s}(t)_{2}\right) \equiv 4(\alpha-k) \gamma(\bmod 16)$. If $\alpha k \equiv 3(\bmod 4)$, then $\operatorname{ord}_{2}\left(d\left(\ell_{s}(t)_{2}\right)\right)$ is odd and hence $\ell_{s}(t)_{2} \rightarrow L_{2}$. Similarly, if $\gamma k \equiv 3(\bmod 4)$, the desired result follows by taking $s \equiv 1$ $(\bmod 2), \operatorname{ord}_{2}(t)=1$. So we may assume that $\alpha k \equiv \gamma k \equiv 1(\bmod 4)$. Consider the two choices of $s, t$ :

$$
s \equiv 1 \quad(\bmod 2), \quad \operatorname{ord}_{2}(t)=0 \quad \text { and } \quad s \equiv 0 \quad(\bmod 2), \quad \operatorname{ord}_{2}(t)=1
$$

Both make $\ell_{s}(t)_{2}$ odd but yield different discriminants over $\mathbb{Q}_{2}$. Therefore one of the two should be represented by $L_{2}$.

Since the remaining subcases can be treated in a very similar manner, we only list the choices of $s$ and $t$ for all possible subcases in Table 3.3 .

REMARK 3.5. As was mentioned in the introduction, $R^{*}(2)=5$ or 6. Although we found an infinite family of quinary positive integral quadratic forms that is contained in $\Re_{5}^{*}(2)$, we could not observe any rule in order for a given quinary form to be a member of $\Re_{5}^{*}(2)$. We could not find a single quinary positive integral quadratic form that is not a member of $\mathfrak{R}_{5}^{*}(2)$, which seems to be the only way to conclude $R^{*}(2)=6$, if this is true. If not, proving $R^{*}(2)=5$ seems to be a very difficult problem. 


\section{Table 3.2}

$$
\begin{array}{ll}
u \geq 2: & \operatorname{ord}_{2}(t)=0 ; \\
u=1, v \geq 1, w \geq 2: & s \equiv 1(\bmod 2), \operatorname{ord}_{2}(t)=0 ; \\
u=1, v \geq 1, w=1: & s \equiv 0(\bmod 2), \operatorname{ord}_{2}(t)=0 ; \\
u=1, v \geq 1, w=0: & \operatorname{ord}_{2}(t)=1 ; \\
u=1, v=0, w \geq 2: & s \equiv 1(\bmod 2), \operatorname{ord}_{2}(t)=1 ; \\
u=1, v=0, w=1: & \operatorname{ord}_{2}(t)=0,2 ; \\
u=1, v=0, w=0: & s \equiv 0(\bmod 2), \operatorname{ord}_{2}(t)=1,2 ; \\
u=0, v \geq 2, w \geq 2: & s \equiv 1(\bmod 2), \operatorname{ord}_{2}(t)=0 ; \\
u=0, v \geq 2, w=1: & \operatorname{ord}_{2}(t)=1 ; \\
u=0, v \geq 2, w=0: & \operatorname{ord}_{2}(t)=2 \text { or } s \equiv 0(\bmod 4), \operatorname{ord}_{2}(t)=1 ; \\
u=0, v=1, w \geq 2: & s \equiv 1(\bmod 2), \operatorname{ord}_{2}(t)=0 ; \\
u=0, v=1, w=1: & \operatorname{ord}_{2}(t)=2 \text { or } s \equiv 1(\bmod 2), \operatorname{ord}_{2}(t)=1 ; \\
u=0, v=1, w=0: & s \equiv 1(\bmod 2), \operatorname{ord}_{2}(t)=0,1 ; \\
u=0, v=0, w \geq 2: & s \equiv 0(\bmod 2), \operatorname{ord}_{2}(t)=1 ; \\
u=0, v=0, w=1: & \operatorname{ord}_{2}(t)=1 \text { or } s \equiv 0(\bmod 2), \operatorname{ord}_{2}(t)=0 ; \\
u=0, v=0, w=0, \operatorname{ord}_{2}\left(\alpha \gamma-\beta^{2}\right) \geq 2: s \equiv 0(\bmod 4), \operatorname{ord}_{2}(t)=0 ; \\
u=0, v=0, w=0, \operatorname{ord}_{2}\left(\alpha \gamma-\beta^{2}\right)=1: \operatorname{ord}_{2}(t)=1 .
\end{array}
$$

\section{Table 3.3}

$u \geq 2, v=0, w \geq 2: \quad s \equiv 0,2(\bmod 4), \operatorname{ord}_{2}(t)=0$

$u \geq 2, v \geq 1, w=1: \quad s \equiv 1(\bmod 2), \operatorname{ord}_{2}(t)=0$;

$u \geq 2, v=0, w=1: \quad \operatorname{ord}_{2}(t)=2$ or $s \equiv 0(\bmod 4), \operatorname{ord}_{2}(t)=0$;

$u \geq 3, v \geq 2, w=0: \quad \operatorname{ord}_{2}(t)=0$

$u \geq 3, v=1, w=0: \quad s \equiv 0,1(\bmod 2), \operatorname{ord}_{2}(t)=0$;

$u \geq 3, v=0, w=0: \quad s \equiv 0(\bmod 2), \operatorname{ord}_{2}(t)=1,2$;

$u=2, v \geq 2, w=0: \quad s \equiv 0,1(\bmod 2), \operatorname{ord}_{2}(t)=0$ 
Table 3.3 (continued)

$$
\begin{aligned}
& u=2, v=1, w=0: \quad \operatorname{ord}_{2}(t)=0 \\
& u=2, v=0, w=0: \quad s \equiv 0(\bmod 2), \operatorname{ord}_{2}(t)=2 \text { or } \\
& s \equiv 0(\bmod 4), \operatorname{ord}_{2}(t)=1 \\
& u=1, v \geq 1, w \geq 2: \quad s \equiv 1(\bmod 2), \text { ord }_{2}(t)=0 \\
& u=1, v \geq 1, w=1: \quad s \equiv 0(\bmod 2), \operatorname{ord}_{2}(t)=0 ; \\
& u=1, v \geq 1, w=0: \quad \operatorname{ord}_{2}(t)=1 \text {; } \\
& u=1, v=0, w \geq 3: \quad s \equiv 0(\bmod 2), \text { ord }_{2}(t)=0 \text {; } \\
& u=1, v=0, w=2: \quad s \equiv \alpha+\beta+3(\bmod 4), \operatorname{ord}_{2}(t)=0 \text { or } \\
& s \equiv 0(\bmod 2), \operatorname{ord}_{2}(t)=0 \\
& u=1, v=0, w=1: \quad \operatorname{ord}_{2}(t)=1 \text {; } \\
& u=1, v=0, w=0: \quad \operatorname{ord}_{2}(t)=2 \text { or } s \equiv 0(\bmod 4), \operatorname{ord}_{2}(t)=1 \text {; } \\
& u=0, v \geq 2, w \geq 3: \quad s \equiv 1(\bmod 2), \operatorname{ord}_{2}(t)=0 ; \\
& u=0, v \geq 2, w=2: \quad \text { see above; } \\
& u=0, v \geq 2, w=1: \quad \operatorname{ord}_{2}(t)=1 \\
& u=0, v \geq 2, w=0: \quad \operatorname{ord}_{2}(t)=2 \text { or } s \equiv 0(\bmod 4), \operatorname{ord}_{2}(t)=1 \text {; } \\
& u=0, v=1, w \geq 4: \quad s \equiv 1(\bmod 2), \operatorname{ord}_{2}(t)=1 \text { or } \\
& s \equiv 2(\bmod 4), \operatorname{ord}_{2}(t)=0 \\
& u=0, v=1, w=3: \quad s \equiv 0(\bmod 4), \operatorname{ord}_{2}(t)=0 \text { or } \operatorname{ord}_{2}(t)=2 ； \\
& u=0, v=1, w=2: \quad s \equiv 1(\bmod 2), \operatorname{ord}_{2}(t)=0 ; \\
& u=0, v=1, w=1: \quad \operatorname{ord}_{2}(t)=1 \text {; } \\
& u=0, v=1, w=0: \quad s \equiv 0,1(\bmod 2), \operatorname{ord}_{2}(t)=1 \text {; } \\
& u=0, v=0, w \geq 2: \quad \operatorname{ord}_{2}(t)=2 \text { or } s \equiv 1(\bmod 2), \operatorname{ord}_{2}(t)=1 \text {; } \\
& u=0, v=0, w=1: \quad \operatorname{ord}_{2}(t)=2 \text { or } s \equiv 0(\bmod 2), \operatorname{ord}_{2}(t)=0 \text {; } \\
& u=0, v=0, w=0, o r d_{2}\left(\alpha \gamma-\beta^{2}\right) \geq 3: s \equiv 0(\bmod 4), o r d_{2}(t)=0 \text {; } \\
& u=0, v=0, w=0, \operatorname{ord}_{2}\left(\alpha \gamma-\beta^{2}\right)=2: s \equiv 1,3(\bmod 4), \operatorname{ord}_{2}(t)=1 \text {; } \\
& u=0, v=0, w=0, \operatorname{ord}_{2}\left(\alpha \gamma-\beta^{2}\right)=1: \quad \operatorname{ord}_{2}(t)=2 \text {. }
\end{aligned}
$$




\section{References}

[1] J. W. S. Cassels, Rational quadratic forms, Academic Press, London-New York, 1978.

[2] W.-K. Chan, M.-H. Kim, and S. Raghavan, Ternary universal integral quadratic forms over real quadratic fields, Japan. J. Math. 22 (1996), 263-273.

[3] J. S. Hsia and M. I. Icaza, Effective version of Tartakowsky's Theorem, Acta Arith. 89 (1999), 235-253.

[4] J. S. Hsia and M. Jöchner, Almost strong approximations for definite quadratic spaces, Invent. Math. 129 (1997), 471-487.

[5] J. S. Hsia, Y. Kitaoka, and M. Kneser, Representations of positive definite quadratic forms, J. Reine. Angew. Math. 301 (1978), 132-141.

[6] D. G. James, Primitive representations by unimodular quadratic forms, J. Number Theory 44 (1993), 356-366.

[7] — Representations by unimodular $\mathbb{Z}$-lattices, Math. Z. 215 (1994), 465475.

[8] M. Jöchner, On the representations of positive definite quadratic forms, Integral Quadratic Forms and Lattices, M.-H. Kim et al (ed.), Contemp. Math. 249 (1999), 73-86.

[9] Y. Kitaoka, Modular forms of degree $n$ and representation by quadratic forms II, Nagoya Math. J. 87 (1982), 127-146.

[10] __ Some remarks on representations of positive definite quadratic forms, Nagoya Math. J. 115 (1989), 23-41.

[11] , The minimum and the primitive representation of positive definite quadratic forms, Nagoya Math. J. 133 (1994), 127-153.

[12] B. M. Kim, M.-H. Kim, and B.-K. Oh, 2-universal positive definite integral quinary quadratic forms, Integral Quadratic Forms and Lattices, M.-H. Kim et al (ed.), Contemp. Math. 249 (1999), 51-62.

[13] M.-H. Kim, J. K. Koo, and B.-K. Oh, Representations of binary forms by certain quinary positive integral quadratic forms, J. Number Theory 89 (2001), 97-113.

[14] B. M. Kim, M.-H. Kim, and S. Raghavan, 2-universal positive definite integral quinary diagonal quadratic forms, Ramanujan J. 1 (1997), 333-337.

[15] M.-H. Kim and B.-K. Oh, A lower bound for the number of squares whose sum represents integral quadratic forms, J. Korean Math, Soc. 33 (1996), 651-655.

[16] _ Representations of positive definite senary integral quadratic forms by a sum of squares, J. Number Theory 63 (1997), 89-100.

[17] B.-K. Oh, Universal $\mathbb{Z}$-lattices of minimal ranks, Proc. Amer. Math. Soc. 128 (2000), 683-689.

[18] Extension of a problem of Kloosterman to lower ranks (preprint).

[19] O. T. O'Meara, Introduction to quadratic forms, Springer-Verlag, New YorkHeidelberg, 1973.

[20] The integral representations of quadratic forms over local fields, Amer. J. Math. 80 (1958), 843-878.

[21] O. Perron, Bemerkungen über die Verteilung der quadratischen Reste, Math. Z. 56 (1952), 122-130.

[22] W. Tartakowsky, Die Gesamtheit der Zahlen, die durch eine positive quadratische Form $F\left(x_{1}, \ldots, x_{s}\right)(s \geq 4)$ darstellbar sind, Isv. Akad. Nauk SSSR 7 
(1929), 111-122, 165-195.

[23] G. L. Watson, Quadratic diophantine equations, Philos. Trans. Roy. Soc. London Ser. A 253 (1960/1961), 227-254.

\section{Myung-Hwan Kim}

Department of Mathematics

Seoul National University

Seoul 151-742, Korea

E-mail: mhkim@math.snu.ac.kr

Byeong-Kweon $\mathrm{Oh}$

Department of Mathematics

Ohio State University

Columbus, Ohio 43210, U.S.A.

E-mail: bkoh@ohio-state.edu 